\title{
Multi-template imprinted polymers for simultaneous selective solid-phase extraction of six phenolic compounds in water samples followed by determination using capillary electrophoresis
}

\author{
Wenhui Lu ${ }^{\mathrm{a}, \mathrm{b}}$, Xiaoyan Wang ${ }^{\mathrm{b}}$, Xiaqing $\mathrm{Wu}^{\mathrm{b}}$, Dongyan Liu ${ }^{\mathrm{b}}$, Jinhua $\mathrm{Li}^{\mathrm{b}}$, Lingxin Chen ${ }^{\mathrm{b}, \mathrm{c}, *}$, \\ Xinshen Zhang ${ }^{\mathrm{a}, * *}$ \\ a National Engineering Laboratory for Clean Technology of Leather Manufacture, Sichuan University, Chengdu 610065, China \\ ${ }^{\mathrm{b}}$ Key Laboratory of Coastal Environmental Processes and Ecological Remediation, Yantai Institute of Coastal Zone Research, Chinese Academy of Sciences, \\ Yantai 264003, China \\ c School of Chemistry and Chemical Engineering, Yantai University, Yantai 264005, China
}

\section{A R T I C L E I N F O}

\section{Article history:}

Received 22 September 2016

Received in revised form 8 December 2016

Accepted 22 December 2016

Available online 23 December 2016

\section{Keywords:}

Multi-template imprinting

Molecularly imprinted polymers

Solid-phase extraction

Capillary electrophoresis

Phenolic compounds

\begin{abstract}
A B S T R A C T
Novel multi-template molecularly imprinted polymers (mt-MIPs) with six phenolic compounds as the template, namely phenol, 4-chlorophenol (4-CP), 2,4,6-trichlorophenol (2,4,6-TCP), 2,4-dichlorophenol (2,4-DCP), 2-chlorophenol (2-CP) and 2,6-dichlorophenol (2,6-DCP), were prepared by precipitation polymerization and used as the adsorbents of solid-phase extraction (SPE), and coupled with capillary electrophoresis (CE) for the simultaneous selective extraction, separation and determination of trace phenolic compounds in water samples. The resultant mt-MIPs exhibited uniform spherical morphology, large specific surface area and high thermal stability, and offered high selectivity towards the six template phenolic compounds. Various parameters affecting the molecularly imprinted SPE (MISPE) efficiency were investigated in detail, and excellent CE separation was realized within $7 \mathrm{~min}$. Good linearity was obtained in the range of $1-200 \mu \mathrm{g} \mathrm{L}^{-1}$ for phenol and $4-\mathrm{CP}$, and $1-300 \mu \mathrm{g} \mathrm{L}^{-1}$ for $2,4,6-\mathrm{TCP}, 2,4-$ DCP, 2-CP and 2,6-DCP. High sensitivity was attained with low limits of detection and quantification ranging from 0.17 to $0.31 \mu \mathrm{g} \mathrm{L}^{-1}$ and 0.57 to $1.03 \mu \mathrm{g} \mathrm{L}^{-1}$, respectively. Satisfactory recoveries for spiked reservoir water, river water, tannery wastewater and tap water samples were achieved in the range of 82.13-105.63\% with relative standard deviations within 1.68-6.96\%. The developed MISPE-CE method proved practically feasible for simultaneous selective extraction/enrichment, separation and sensitive determination of multiple targets in complicated aqueous matrices.
\end{abstract}

(c) 2016 Elsevier B.V. All rights reserved.

\section{Introduction}

Sample preparation plays an important part in eliminating matrix effect and enriching the trace amount of target analytes, which is also a time-consuming step for the whole analytical procedure. Therefore, developing selective, reliable, simple, easily automatic and time-saving pretreatment techniques are urgently required. Phenolic compounds especially chlorinated ones are toxic and persistent in environment with low concentration, which have

\footnotetext{
* Corresponding author at: Key Laboratory of Coastal Environmental Processes and Ecological Remediation, Yantai Institute of Coastal Zone Research, Chinese Academy of Sciences, Yantai 264003, China.

** Corresponding author.

E-mail addresses: lxchen@yic.ac.cn (L. Chen), zhangxinshen@126.com (X. Zhang).
}

been included in the list of priority pollutants devised by European Union and United State Environmental Protection Agent [1]. For the enrichment of trace/ultra-trace level phenolic compounds, many kinds of traditional and novel pretreatment techniques have been proposed, such as, cloud point extraction [2], liquid phase microextraction [3], dispersive liquid-liquid microextraction [4], solid-phase extraction (SPE) [5], magnetic extraction [6], solidphase microextraction [7] and stir-bar sorptive extraction [8]. Among them, SPE as a convenient and easy to automate technique has been widely used in environment, food and biological fields [9]. The commercially available SPE cartridges mainly are packed with silica, $\mathrm{C}_{18}, \mathrm{C}_{8}$, florisil and $\mathrm{Al}_{2} \mathrm{O}_{3}$. For the commonly used SPE sorbents, target analytes are usually retained on their functionalized surface by physicochemical interaction. Besides the target, other matrix compounds can also be retained on SPE sorbents unavoidably. Thus, many novel materials with the performance of high 
selectivity and stability have been utilized for SPE [9], for example, molecularly imprinted polymers (MIPs) with artificial recognition cavities complementary to the template molecules in shape, size and chemical functionality $[9,10]$, which can specifically rebind the template molecules from the complicated matrices [10].

MIPs, with the unique features of structure predictability, recognition specificity and application universality, have found widespread applications in sample pretreatment, chromatographic separation and chemical/biological sensing [11]. In particular, the use of MIPs as SPE sorbents is one of the most commonly applications in sample pretreatment techniques, called molecularly imprinted SPE (MISPE) [11], which can provide cleaner sample extract than usual SPE. To synthesize MIPs suitable for SPE, several kinds of preparation methods have been proposed including bulk polymerization, precipitation polymerization, suspension polymerization, emulsion polymerization, surface imprinting and so on, and relevant studies on phenols and chlorophenols have been increasingly reported [10-14]. For instances, El-Sheikh et al. used 2-chlorophenol (2-CP) as template to synthesize MIPs by bulk polymerization and applied the MIPs to MISPE coupled with highperformance liquid chromatography (HPLC) for the enrichment and determination of 2-CP in water sample [12]. Mohamad et al. prepared MIPs by bulk polymerization using 2,4-dichlorophenol (2,4-DCP) as template for MISPE of phenols in water samples [13]. The obtained MIPs by bulk polymerization should be ground and sieved before usage, with irregular morphology and heterogeneous size distribution. Lin et al. synthesized MIPs by precipitation polymerization using 2,4,6-trichlorophenol (2,4,6-TCP) as template and used as micro-SPE procedure for the selective pre-concentration of phenolic compounds from environmental water samples [14]. In most of the molecular imprinting, a single template molecule is used to create the specific binding site of the MIPs; however, the imprinting process does not have to be limited to a single template, and several templates can be imprinted simultaneously [15]. Multi-template imprinting, using dual/multiple targets/species as templates, can provide multiple types of recognition sites in one MIPs format, which can widen the application of MIPs for more than one analyte $[11,15,16]$. And several different classes of templates can be recognized, extracted, separated, detected, or otherwise analyzed at one time [15], which will be highly desirable for simultaneously monitoring a group of possible contaminants in environmental systems.

Therefore, in the present work, we adopted multi-template imprinting strategy to synthesize novel MIPs (mt-MIPs) by using the mixture of six phenolic compounds as templates including phenol, 2-CP, 4-chlorophenol (4-CP), 2,4-DCP, 2,6-dichlorophenol (2,6-DCP) and 2,4,6-TCP, in order to simultaneously highly selectively extract the six compounds from water samples, followed by the determination using capillary electrophoresis (CE) owing to its high resolving power, rapidity, and low samples/reagents consumption. Coupling MISPE with CE combines highly selective cleanup and enrichment technique with a highly efficient separation technique, and can offer an ideal alternative for simultaneous analysis of multiple targets in complex samples [17-19]. In order to investigate the more suitable MISPE sorbents, two simple and widely used polymerization methods of bulk polymerization and precipitation polymerization were used. The as-prepared mtMIPs were fully characterized and evaluated by scanning electron microscopy (SEM), Brunauer-Emmett-Teller (BET), Fourier transform infrared spectroscopy (FT-IR) and thermogravimetric analysis (TGA). As well as, their adsorption properties were studied. Consequently, main parameters affecting the MISPE efficiency were investigated systematically, including sample $\mathrm{pH}$, type and volume of elution solvent, flow rate of loading sample and salt effect. The validated MISPE-CE method demonstrated excellent linearity, precision and accuracy, and was successfully applied to real water samples, indicating great potentials for high throughput extraction and analysis.

\section{Experimental}

\subsection{Reagents and materials}

HPLC grade reagents of phenol, 2-chlorophenol (2-CP), 4-chlorophenol (4-CP), 2,4-dichlorophenol (2,4-DCP) and 2,4,6trichlorophenol (2,4,6-TCP) were obtained from Sigma-Aldrich (Shanghai, China) and the standard stock solutions were prepared by dissolving them in methanol of a concentration of $1000 \mathrm{mg} \mathrm{L}^{-1}$. The standard solution of 2,6-dichlorophenol (2,6-DCP) in methanol with concentration of $1000 \mathrm{mg} \mathrm{L}^{-1}$ was purchased from J\&K Scientific (Shanghai, China). 3-Chlorophenol (3-CP), 4-chloro-3methylphenol (PCMC), 4-nitrophenol (4-NP) and 2-nitrophenol (2-NP) were purchased from Aladdin (Shanghai, China). All the standard solutions were stored at $4{ }^{\circ} \mathrm{C}$ in a refrigerator and used for $\mathrm{CE}$ separation. Sodium tetraborate decahydrate $\left(\mathrm{Na}_{2} \mathrm{~B}_{4} \mathrm{O}_{7} \cdot 10 \mathrm{H}_{2} \mathrm{O}\right)$ and sodium hydroxide $(\mathrm{NaOH})$ were supplied from Sinopharm Chemical Reagent Co. Ltd. (Shanghai, China).

Methacrylic acid (MAA) and divinylbenzene (DVB) were purchased from Sigma-Aldrich and purified prior to use in order to remove stabilizers. 2,2'-Azo-bis-isobutyronitrile (AIBN) was attained from Aladdin (Shanghai, China) and recrystallized in $50^{\circ} \mathrm{C}$ ethanol prior to use. Acetonitrile, methanol, toluene and acetic acid were obtained by Sinopharm Chemical Reagent Co., Ltd. (Shanghai, China).

Ultrapure water with the specific resistances of $18.2 \mathrm{M} \Omega \mathrm{cm}$ was produced by Pall cascada ${ }^{\mathrm{TM}}$ lab water purification system (Pall Corp., USA) for aqueous solution preparation throughout the study. All other solvents and chemicals were of analytical grade and used directly without further purification.

\subsection{Instrumentation}

CE measurements were carried out on a Beckman P/ACE ${ }^{\mathrm{TM}} \mathrm{MDQ}$ Capillary Electrophoresis System (USA) equipped with a diode array detector (DAD). The detection wavelength was set at $195 \mathrm{~nm}$ for phenol, 4-CP and 2-CP, $214 \mathrm{~nm}$ for 2,4,6-TCP, 2,4-DCP and 2,6DCP. The capillary temperature was maintained at $25^{\circ} \mathrm{C}$ and the applied voltage was $+20 \mathrm{kV}$ and pressure injection was performed using 0.5 psi for $5 \mathrm{~s}(1 \mathrm{psi}=6894.76 \mathrm{~Pa})$. Bare fused-silica capillary with $50.2 \mathrm{~cm}$ total length, $40 \mathrm{~cm}$ effective length, and $75 \mu \mathrm{m}$ i.d. was used as separation column (Yongnian Optical Fiber Co. Ltd., Hebei, China). The pH value measurements were made with a Rex pH meter (Shanghai Precision Scientific Instrument Corporation, Shanghai, China). New capillary was preconditioned by rinsing with methanol for $5 \mathrm{~min}$, water for $5 \mathrm{~min}, 1 \mathrm{~mol} \mathrm{~L}^{-1} \mathrm{NaOH}$ for $30 \mathrm{~min}$, water for $10 \mathrm{~min}$ and running buffer for $30 \mathrm{~min}$. Each day, the capillary was conditioned by flushing with water for $5 \mathrm{~min}, 1 \mathrm{~mol} \mathrm{~L}^{-1}$ $\mathrm{NaOH}$ for $5 \mathrm{~min}$ and then water for $5 \mathrm{~min}$. Between each run, the capillary was rinsed with separation buffer for $5 \mathrm{~min}$, which consisted of $20 \mathrm{mmol} \mathrm{L}^{-1}$ borate buffer containing $10 \%(\mathrm{v} / \mathrm{v})$ acetonitrile, and its $\mathrm{pH}$ was adjusted after adding acetonitrile to 9.8 using $1 \mathrm{~mol} \mathrm{~L}^{-1} \mathrm{NaOH}$. All electrolytes and samples were filtered through $0.22 \mu \mathrm{m}$ membrane filters and degassed by ultrasonic prior to analysis.

For characterization of polymers, scanning electron microscope (SEM, Hitachi S-4800, Japan) was used for morphological evaluation. All samples were sputter-coated with gold before SEM analysis. Specific surface area was measured by nitrogen sorption porosimetry performed on Full-automatic Specific Surface Instruments (3H-2000PS4, Beishide Instrument Technology, Beijing) and calculated by Brunauer-Emmett-Teller (BET) method. 
Size distribution was determined by a laser particle analyzer (Marlvern Mastersizer 2000F, UK). Fourier transform infrared (FT-IR) spectrometer (Thermo Nicolet Corporation, USA) was employed to examine the infrared spectra of sample using a pressed $\mathrm{KBr}$ tablet. The wavenumbers of FT-IR measurement range were controlled from 400 to $4000 \mathrm{~cm}^{-1}$. The thermal stability of polymers was tested by thermogravimetric analysis (TGA) using a Mettler 5MP/PF7548/MET/400W thermal analyzer in nitrogen atmosphere (Mettler Toledo, Switzerland), presenting TGA and derivative thermogravimetry (DTG) data.

\subsection{Preparation of $m t-M I P s$}

The mt-MIPs were prepared according to noncovalent approach by bulk polymerization and precipitation polymerization, respectively. The obtained imprinted and non-imprinted polymers (NIPs) were named BPMIPs and BPNIPs for bulk polymerization, PPMIPs and PPNIPs for precipitation polymerization, respectively.

For the preparation of BPMIPs, template compounds containing phenol, 2-CP, 4-CP, 2,4-DCP, 2,6-DCP and 2,4,6-TCP with molarity of $0.2 \mathrm{mmol}$ individual were dissolved in $4 \mathrm{~mL}$ acetonitrile/toluene $(3: 1, v / v)$ in a $20 \mathrm{~mL}$ glass vial and $4.8 \mathrm{mmol}$ MAA (monomer) was added to the solution. After the $12 \mathrm{~h}$ pre-polymerization process at $4{ }^{\circ} \mathrm{C}, 24 \mathrm{mmol}$ DVB (cross-linker) and $40 \mathrm{mg}$ AIBN (initiator) were added to the mixture solution. Then, the above solution was deoxygenated with nitrogen for $15 \mathrm{~min}$ to remove dissolved oxygen and the vial was sealed under a nitrogen atmosphere and placed in water bath. The temperature of water bath ramped from room temperature to $60^{\circ} \mathrm{C}$ around $2 \mathrm{~h}$ and kept at this temperature for $24 \mathrm{~h}$ to polymerize. After the polymerization, the resultant polymers were crushed, ground and washed with acetonitrile/acetic acid (90:10, $\mathrm{v} / \mathrm{v}$ ) in a Soxhlet extraction system to remove template molecules until no template was detected by CE. To remove acetic acid, acetonitrile was used to wash the polymers. Then, the obtained BPMIPs were dried in a vacuum drying oven at $40^{\circ} \mathrm{C}$ for use. As a control, the corresponding BPNIPs were also synthesized using the same procedure only without template molecules.

The preparation procedure of precipitation polymerization is similar to bulk polymerization but with the addition of larger amounts of porogen, as schematically shown in Fig. 1A. For precipitation polymerization, template compounds containing phenol, 2-CP, 4-CP, 2,4-DCP, 2,6-DCP and 2,4,6-TCP with molarity of $0.1 \mathrm{mmol}$ individual were dissolved in $80 \mathrm{~mL}$ acetonitrile/toluene $(3: 1, v / v)$ in a $250 \mathrm{~mL}$ glass flask and $2.4 \mathrm{mmol}$ MAA (monomer) was added to the solution. After the $12 \mathrm{~h}$ pre-polymerization process at $4{ }^{\circ} \mathrm{C}, 12 \mathrm{mmol}$ DVB (cross-linker) and $20 \mathrm{mg}$ AIBN (initiator) were added to the mixture solution. Then, the above solution was deoxygenated with nitrogen for 15 min to remove dissolved oxygen and the flask was sealed under a nitrogen atmosphere and placed in water bath. The temperature of water bath ramped from room temperature to $60^{\circ} \mathrm{C}$ around $2 \mathrm{~h}$ and kept at this temperature for $24 \mathrm{~h}$ to polymerize. After the polymerization, with the help of centrifugation, the resultant polymers were washed with acetonitrile to remove the unreacted reagents and then washed with acetonitrile/acetic acid $(90: 10, v / v)$ to remove the template molecules until no template was detected by $C E$. After washing with acetonitrile to remove acetic acid, the obtained PPMIPs were dried in a vacuum drying oven at $40^{\circ} \mathrm{C}$ for use. As a control, the corresponding PPNIPs were also synthesized using the same procedure only without template molecules.

\subsection{Adsorption experiments}

To evaluate the static adsorption capacity of the obtained polymers to six phenolic compounds, 2 mg MIPs (BPMIPs or PPMIPs) or NIPs (BPNIPs or PPNIPs) were added into $4 \mathrm{~mL}$ aqueous solu- tion of six phenolic compounds with various concentrations from 10 to $300 \mathrm{mg} \mathrm{L}^{-1}$ in $10 \mathrm{~mL}$ centrifuge tubes. Then the mixture was sealed and shaken for $24 \mathrm{~h}$ at room temperature to ensure the equilibrium. After centrifugation and filtration with $0.22 \mu \mathrm{m}$ cellulose acetate membrane filters, the solution containing the free template compounds was measured by $C E$. The adsorption capacity $(Q)$ was calculated according to Eq. (1):

$Q=\frac{\left(C_{0}-C_{1}\right) V}{m}$

where $C_{0}$ and $C_{1}$ are the concentration of template compounds individual in solution before and after adsorption, $V$ is the volume of the solution, and $m$ is the mass of MIPs or NIPs.

In order to estimate the selectivity of mt-MIPs for the six template compounds, other four similar compounds of 3-CP, PCMC, 4-NP and 2-NP were chosen and investigated. Briefly, 2 mg PPMIPs or PPNIPs were dispersed in $4 \mathrm{~mL}$ of aqueous solution of the six template compounds, and mixed with 3-CP, PCMC, 4-NP and 2-NP with the concentration of $200 \mathrm{mg} \mathrm{L}^{-1}$ individual in $10 \mathrm{~mL}$ centrifuge tubes, respectively. The mixture was shaken for $24 \mathrm{~h}$ at room temperature, followed by centrifugation and filtration with $0.22 \mu \mathrm{m}$ cellulose acetate membrane filters before detected by CE. The specific recognition ability of mt-MIPs was evaluated by the imprinting factor (IF), which was calculated by Eq. (2),

$\mathrm{IF}=\frac{Q_{\mathrm{MIP}}}{Q_{\mathrm{NIP}}}$

where $Q_{\text {MIP }}$ and $Q_{\text {NIP }}$ are the adsorption capacity of the template or their analogues on mt-MIPs and mt-NIPs, respectively.

The distribution coefficients $\left(K_{\mathrm{d}}\right)$, selectivity coefficients $(K)$ and relative selectivity coefficient $\left(K^{\prime}\right)$ of six phenolic compounds with respect to 3-CP, PCMC, 4-NP and 2-NP were also utilized to evaluate the selectivity of mt-MIPs [20]. Distribution coefficient $\left(K_{\mathrm{d}}\right)$ was calculated by Eq. (3),

$K_{\mathrm{d}}=\frac{Q_{\mathrm{e}}}{C_{\mathrm{e}}}$

where $K_{\mathrm{d}}$ represents the distribution coefficient $\left(\mathrm{mLg}^{-1}\right), Q_{\mathrm{e}}$ $\left(\mathrm{mg} \mathrm{g}^{-1}\right)$ is the equilibrium adsorption capacity, $C_{\mathrm{e}}\left(\mathrm{mg} \mathrm{L}^{-1}\right)$ is the equilibrium concentration.

The selectivity coefficient $(K)$ of mt-MIPs was calculated by Eq. (4),

$K=\frac{K_{\mathrm{d}(\mathrm{tem})}}{K_{\mathrm{d}(\mathrm{ana})}}$

where $K_{\mathrm{d}(\mathrm{tem})}$ and $K_{\mathrm{d}(\mathrm{ana})}$ are the distribution coefficients of template and structurally related compounds, respectively.

The relative selectivity coefficient $\left(K^{\prime}\right)$ was defined as Eq. (5), which indicated the enhanced extent of adsorption affinity and selectivity of template molecule on mt-MIPs with respect to mtNIPs,

$K^{\prime}=\frac{K_{\mathrm{MIP}}}{K_{\mathrm{NIP}}}$

where $K_{\mathrm{MIP}}$ and $K_{\mathrm{NIP}}$ are the selectivity coefficient of mt-MIPs and mt-NIPs, respectively, the higher value of $K^{\prime}$, the greater the difference between mt-MIPs and mt-NIPs.

\subsection{MISPE procedure and sample preparation}

For the MISPE study, $60 \mathrm{mg}$ MIPs or NIPs were packed into $3 \mathrm{~mL}$ empty polyethylene cartridge between two polyethylene frits with $2 \mu \mathrm{m}$ in porosity size (Biocomma, Shenzhen, China). The packed cartridges were connected to a SPE vacuum manifold (Agela Technologies, Tianjin, China) and the schematic procedure was shown in Fig. 1B. The cartridges were firstly conditioned with $3 \mathrm{~mL}$ acetonitrile/acetic acid $(9: 1, v / v), 6 \mathrm{~mL}$ acetonitrile and $3 \mathrm{~mL}$ water. Then, 


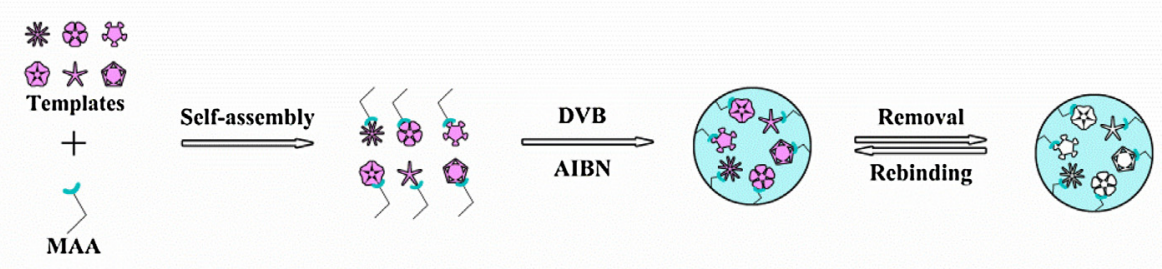

B
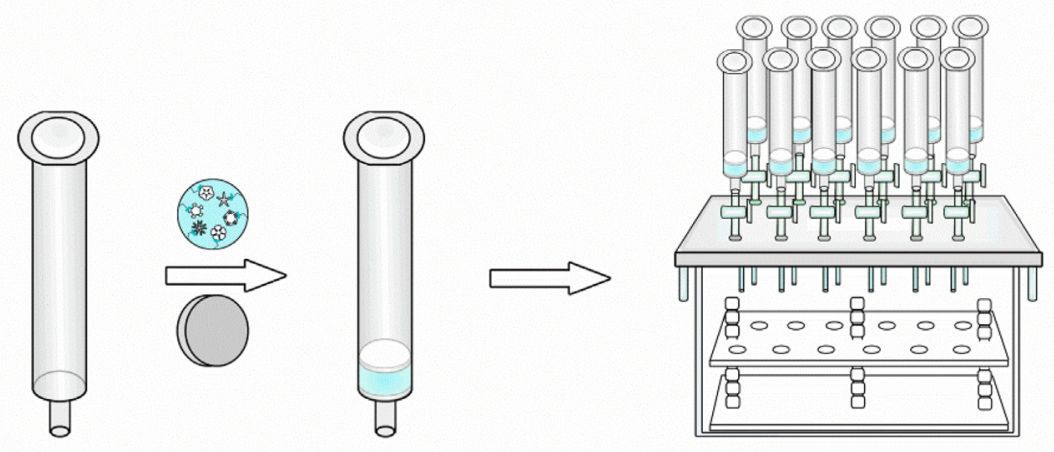

\section{C}
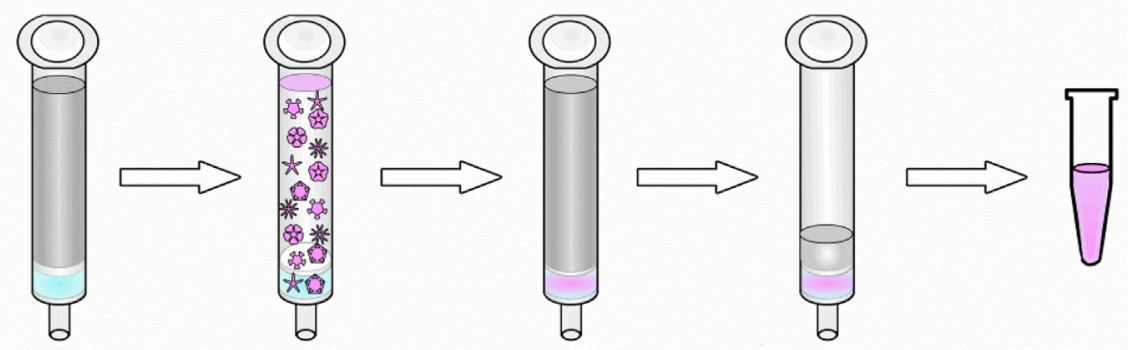

Conditioning Loading

Washing

Eluting
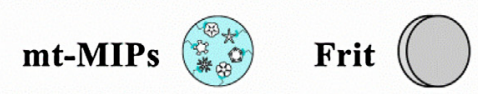

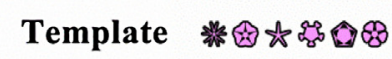

Fig. 1. Schematic procedures for the preparation of mt-MIPs by precipitation polymerization (A), packing of MISPE cartridge (B) and MISPE (C).

$15 \mathrm{~mL}$ aqueous solution containing suitable concentration of six phenolic compounds was loaded onto the cartridges with the speed of $1 \mathrm{~mL} \mathrm{~min}^{-1}$. Subsequently, the cartridges were washed with $3 \mathrm{~mL}$ water and dried for 5 min by passing air. The retained phenolic compounds were eluted with $400 \mu \mathrm{L}$ acetonitrile containing 1\% acetic $\operatorname{acid}(\mathrm{v} / \mathrm{v})$ which was collected for CE. The schematic procedure was shown in Fig. 1C.

Reservoir and river water samples were collected from the coastal zone area of Yantai City (China), tannery wastewater was collected from our laboratory in Sichuan University (Chengdu City, China), and all these water samples were stored at $4{ }^{\circ} \mathrm{C}$ in a refrigerator before analysis. Tap water was collected after it had flowed for about $5 \mathrm{~min}$ in the laboratory when needed. Before use, all the water samples were filtered through a $0.22 \mu \mathrm{m}$ filter to remove possible suspended particles.

\section{Results and discussion}

\subsection{Preparation and characterization of $m t-M I P S$}

In order to obtain more suitable adsorbents of MISPE for selective adsorption of phenolic compounds, two kinds of mt-MIPs by bulk polymerization and precipitation polymerization were synthesized. The choice of monomer, porogen and cross-linker plays an important part role in the preparation of MIPs with homogeneous morphology, excellent adsorption and recognition properties. MAA was chosen as functional monomer because the hydrogen bond can be formed between carboxyl acid group of MAA and phenolic hydroxyl group of phenolic compounds. In order to obtain large particles MIPs with controllable morphology for SPE, matching the solubility parameter of the developing polymer network to that of the porogenic solvent(s) is particularly important, which is usually met for the copolymerization of DVB in the mixture of acetonitrile and toluene [21]. Therefore, DVB was chosen as cross-linker and acetonitrile/toluene $(3: 1, v / v)$ as porogen, which could prepare the polymers with larger average diameters and specific surface area.

SEM images of the prepared mt-MIPs and NIPs were shown in Fig. 2A. Compared with BPMIPs and BPNIPs with irregular morphology (Fig. 2A(a), (b)), PPMIPs and PPNIPs were uniform spherical particles (Fig. 2A(c), (d)) with larger average diameters. The size distributions of PPMIPs and PPNIPs were shown in Fig. 2B. As seen, the intensity/volume contribution versus diameters of particles exhibited a good size distribution and a dominant distribution peak around $4 \mu \mathrm{m}$ for the PPMIPs and $700 \mathrm{~nm}$ for PPNIPs. Obvi- 


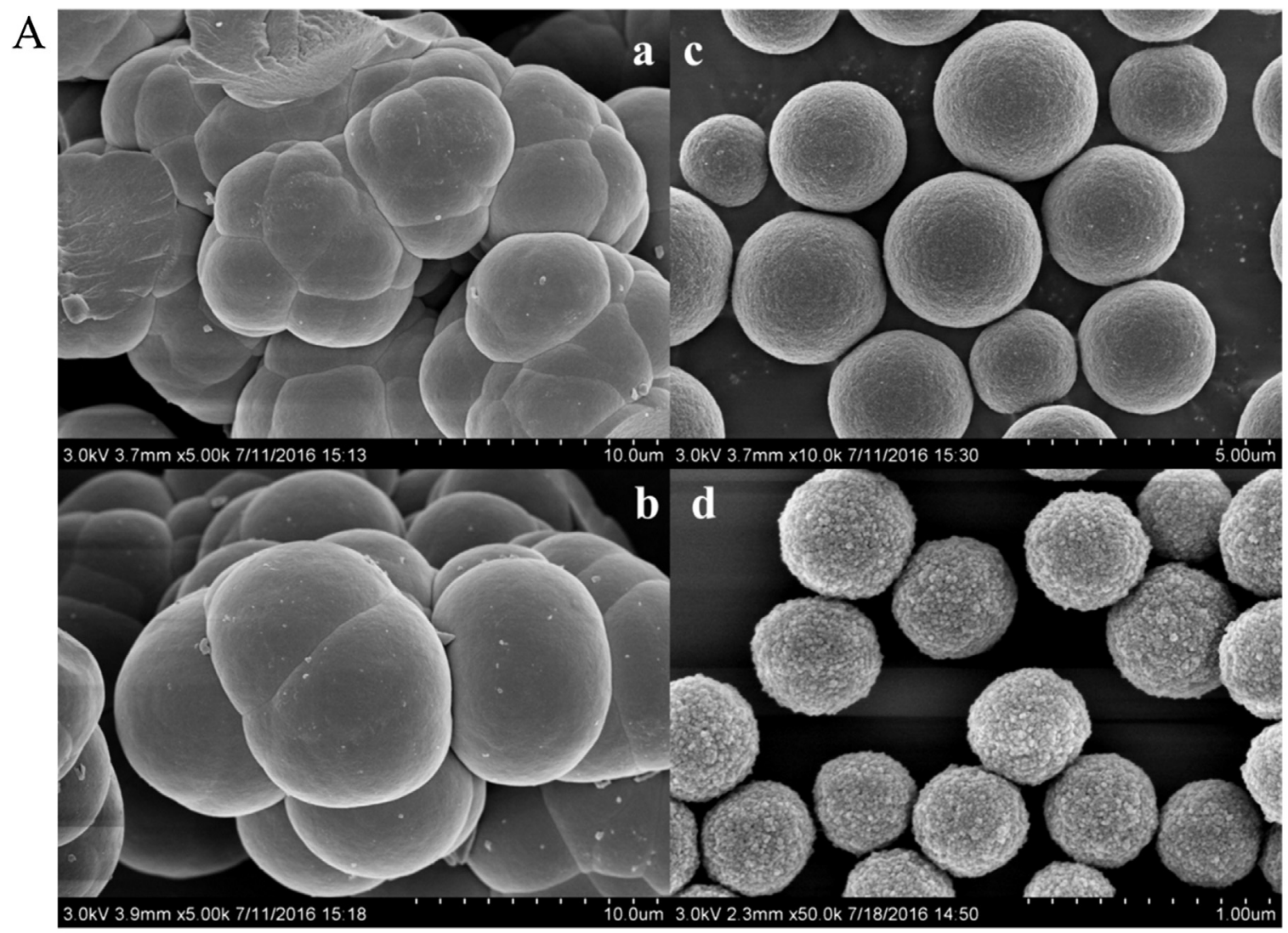

B

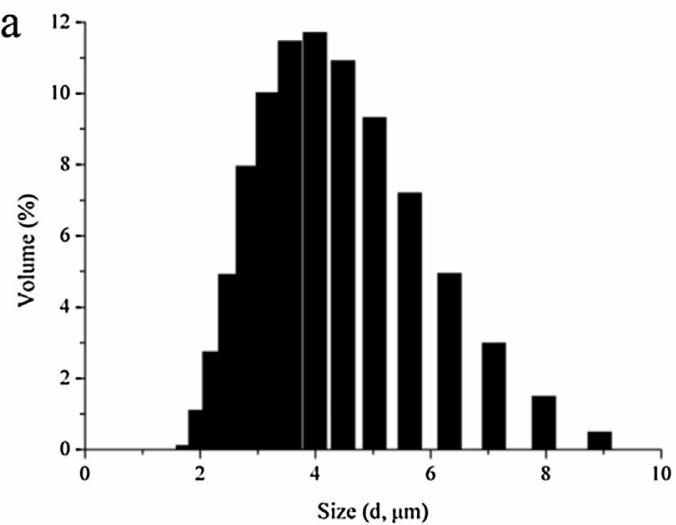

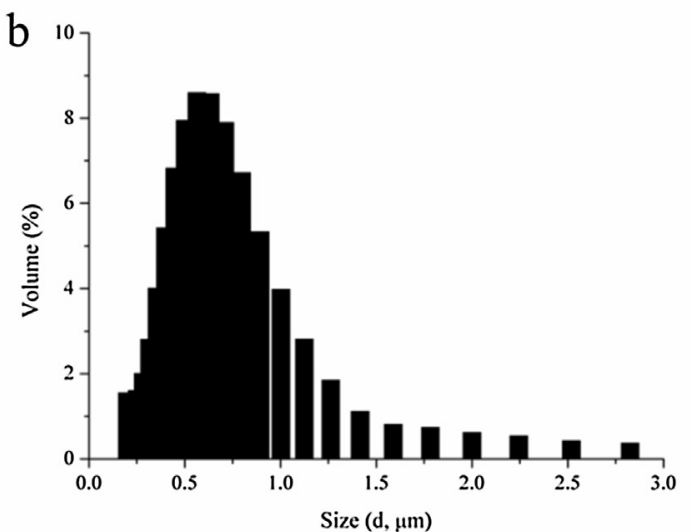

Fig. 2. (A) SEM images of BPMIPs (a), BPNIPs (b), PPMIPs (c) and PPNIPs (d). (B) Particle size distribution profiles of PPMIPs (a) and PPNIPs (b).

ously, the size of PPMIPs was larger than that of PPNIPs, possibly owing to the influence of templates on the nucleation stage of precipitation polymerization and thereby suggesting the important role of imprinting. Moreover, the BET analytical results of MIPs and NIPs were attained. As listed in Table S1, the PPMIPs possessed much larger specific surface area $\left(758.52 \mathrm{~m}^{2} \mathrm{~g}^{-1}\right)$ than BPMIPs $\left(481.07 \mathrm{~m}^{2} \mathrm{~g}^{-1}\right)$, which could be attributed to the spherical morphology of PPMIPs, and would lead to higher adsorption capacity.

FT-IR spectra of the PPMIPs before and after template compounds removal were shown in Fig. 3A. Strong absorption bands at around 3432, 2930 and $1700 \mathrm{~cm}^{-1}$ were observed, which could be attributed to the stretching vibration of $\mathrm{C}=\mathrm{O}, \mathrm{C}-\mathrm{H}$ and $\mathrm{O}-\mathrm{H}$ in MAA [22]. Absorption bands at around 1600 and $1450 \mathrm{~cm}^{-1}$ were observed due to $C=C$ stretching vibration in benzene ring of DVB or MAA [23]. The bonds at around 1099 and $1160 \mathrm{~cm}^{-1}$ were attributed to $\mathrm{C}-\mathrm{O}$ stretching and $\mathrm{O}-\mathrm{H}$ bending vibrations
[24]. Three bonds at 833,798 and $709 \mathrm{~cm}^{-1}$ were observed corresponding to the out-of-plane bending of the aromatic $\mathrm{C}-\mathrm{H}$ [25], which became weak after removal of template compounds. Moreover, the disappearance of $\mathrm{C}-\mathrm{Cl}$ stretching vibration at $590 \mathrm{~cm}^{-1}$ (curve b) [26] also indicated the successful imprinting of phenolic compounds.

The thermal stability of PPMIPs was tested by TGA, which measured the weight change of polymers by programmed temperature/time profile [27]. As shown in Fig. 3B, the weight of PPMIPs increased slightly with the temperature increasing from $30^{\circ} \mathrm{C}$ to $380^{\circ} \mathrm{C}$. One of the possible reasons is that the rate of weight loss of PPMIPs was slow and the PPMIPs were very stable below $380^{\circ} \mathrm{C}$. The other possible reason is the pore structure of PPMIPs can adsorb a small amount of nitrogen in the TGA procedure [28]. At the temperature ranging from $380^{\circ} \mathrm{C}$ to $480^{\circ} \mathrm{C}$, a high rate of weight loss (close to 68.66\%) appeared possibly resulting from the decomposition of cross-linker (DVB). These observations can also be clearly 
A
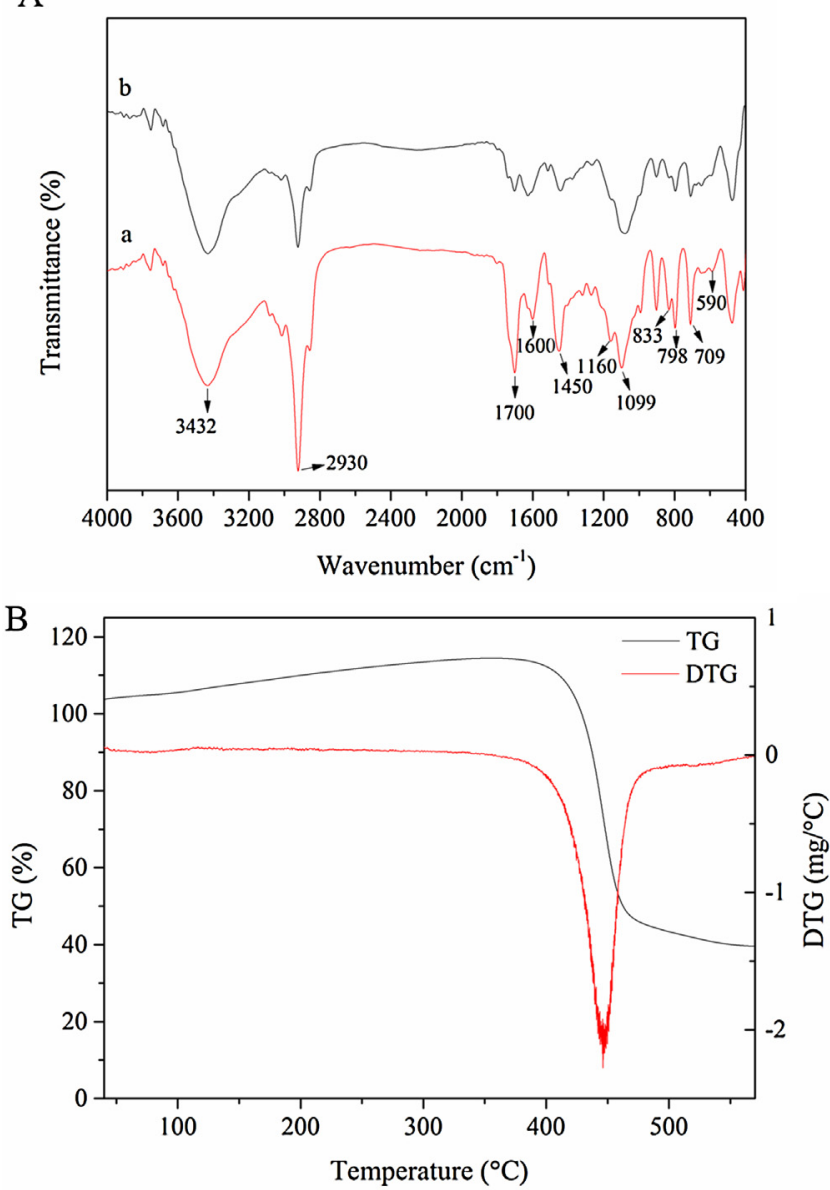

Fig. 3. (A) FT-IR spectra of PPMIPs before (a) and after (b) templates adsorption. (B) TG/DTG curves of PPMIPs.

shown from DTG curve (Fig. 3B). Hence, the prepared mt-MIPs by precipitation polymerization demonstrated good thermal stability at temperature lower than $380^{\circ} \mathrm{C}$.

\subsection{Adsorption analysis and recognition selectivity of mt-MIPs}

The adsorption experiments of mt-MIPs/NIPs were carried out and the adsorption isotherms were shown in Fig. S1. As observed, high adsorption capacities of PPMIPs and BPMIPs for the six templates compounds were attained, in the range of $130-400 \mathrm{mg} \mathrm{g}^{-1}$ and $97-241 \mathrm{mg} \mathrm{g}^{-1}$, respectively. For both PPMIPs and BPMIPs, the adsorption capacity of six phenolic compounds increased fast with the increasing of the initial concentration and then tended to stable at high concentration, while their corresponding NIPs had the same trends and relatively lower binding capacity. Based on adsorption-desorption equilibrium between polymers and template compounds, at low concentration, a small number of recognition sites of MIPs were occupied by adsorbed template compounds resulting in low adsorption capacity; with the increasing of concentration, the adsorption capacity increased sharply and reached saturated adsorption, ensuring almost all recognition sites were occupied. Except MIPs showed high binding affinity for templates, NIPs had lower adsorption capacity because of the absence of specific recognition sites. According to the results, the multitemplate imprinting, using six compounds as templates, provided multiple recognition sites in one MIPs format, and thus the synthesized mt-MIPs could highly selectively recognize and bind the six phenolic compounds simultaneously. Such high adsorption capac- ities would offer high efficient enrichment and thereby facilitate (ultra)trace analysis. Besides, the adsorption capacities of PPMIPs were significantly higher than that of BPMIPs, possibly attributing to the spherical morphology and larger specific surface area of PPMIPs. Based on the above results, PPMIPs proved more suitable sorbents for MISPE of phenolic compounds than BPMIPs.

Furthermore, the selective recognition ability of mt-MIPs was evaluated by investigating the adsorption capacities of PPMIPs and PPNIPs towards the six template compounds and their structurally similar compounds including 3-CP, PCMC, 4-NP and 2-NP. As seen from Fig. S2, PPMIPs and PPNIPs showed similar low adsorption capacities for 3-CP, PCMC, 4-NP and 2-NP, which indicated that the interaction with these compounds occurred by non-specific adsorption in the polymers. In contrast, adsorption capacities of the PPMIPs were much higher for the six templates, because a larger number of specific imprinting/recognition sites complementary with the template molecules in shape, size, and functionality were generated on the mt-MIPs in the synthesis process. Hence, the templates could be strongly rebound to the mt-MIPs and then produce significantly high adsorption capacities. Besides, imprinting factor (IF), distribution coefficient $\left(K_{\mathrm{d}}\right)$, selectivity coefficient $(K)$ and relative selectivity coefficient $\left(K^{\prime}\right)$ of templates with respect to the analogues on mt-MIPs and mt-NIPs were measured for the selectivity evaluation, summarized in Table S2. As seen from the table, the IF of six template compounds were obtained above 2.02, but only 1.38 for 3-CP, 1.23 for PCMC, 1.16 for 4-NP and 1.23 for $2-\mathrm{NP}$, indicating the good imprinting effect. The $K_{\mathrm{d}}$ values of six templates on mt-MIPs were greater than those of mt-NIPs, which could be attribute to the random distribution of ligand functionalities in the polymeric network of mt-NIPs. Interestingly, there are considerable amounts of imprinted cavities/recognition sites with a predetermined orientation and special size in the mt-MIPs, leading to ordered distribution and excellent imprinting efficiency [20]. The $K$ values of mt-MIPs for six phenolic compounds with respect to 3-CP, PCMC, 4-NP and 2-NP increased after imprinted; the phenomenon implied that the adsorption of NIPs for template and structurally related compounds lacked selectivity [29]. The possible reason is that the analogues could not match to the imprinted cavities of mt-MIPs in size, shape and spatial arrangement. Especially for phenol, the competitive compounds are larger than phenol in size, so they could not enter into the corresponding cavities imprinted by phenol and difficult to form hydrogen bond with mt-MIPs [30]. The $K^{\prime}$ value is an indicator to express the enhancement of adsorption affinity and selectivity of mt-MIPs for template versus mt-NIPs. Its related results indicated that the geometric affinity also played an essential role in the selectivity of MIPs except the hydrogen bond ability between mt-MIPs and template [31]. Consequently, all the above results from Fig. S2 and Table S2 suggested the mt-MIPs could be used for the simultaneous selective recognition/adsorption of the six targeted templates.

\subsection{Condition optimization of the mt-MIPs based MISPE procedure}

According to the above characterization results, the PPMIPs with larger specific surface and higher binding capacity could be selected as the adsorbents of SPE, coupled with CE-DAD for enrichment and detection six phenolic compounds in aqueous solutions. Similar with the traditional SPE method, MISPE procedure also include conditioning, loading, washing and eluting. Firstly, the MISPE cartridges were washed with acetonitrile/acetic acid $=9: 1(\mathrm{v} / \mathrm{v})$ and acetonitrile to remove any residual template compounds that could potentially interfere with the further analysis, until no template was detected by CE. In order to obtain the maximum extraction efficiency, the MISPE procedure was optimized by evaluating sample $\mathrm{pH}$, type and volume of elution solvent, flow rate of loading 
A
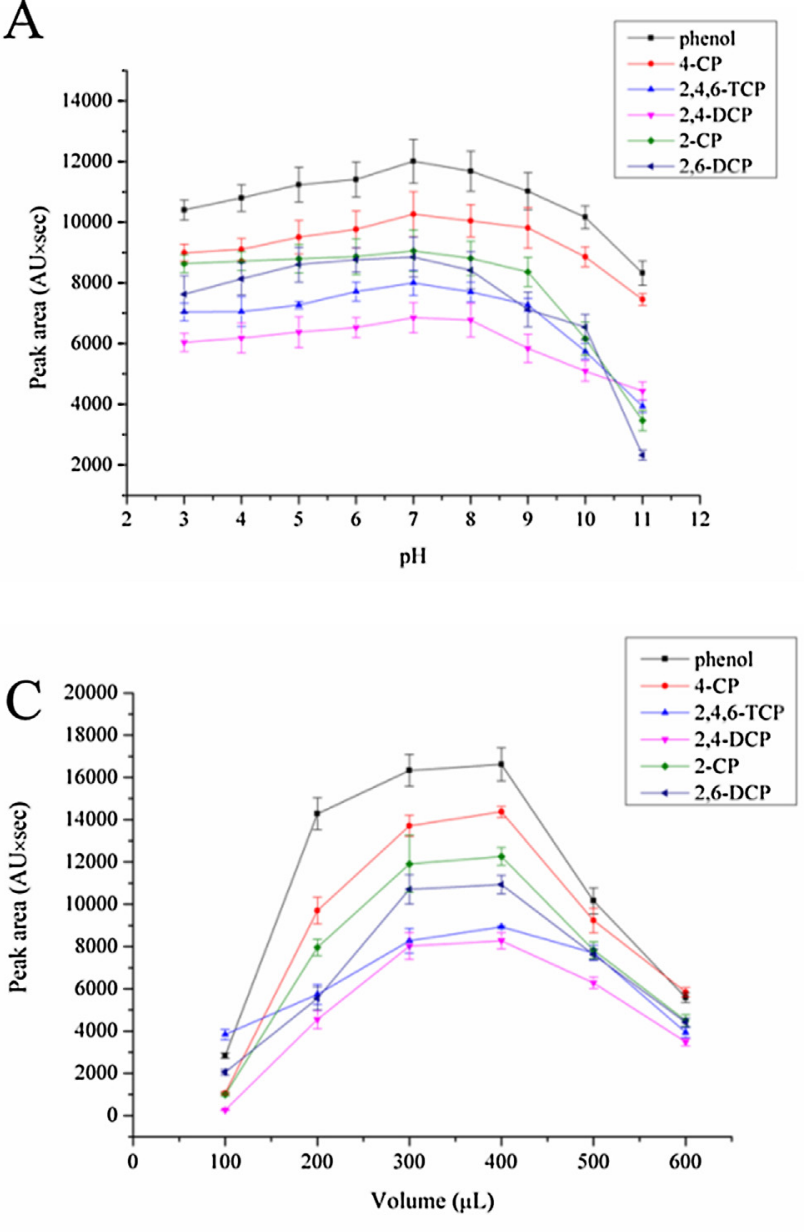
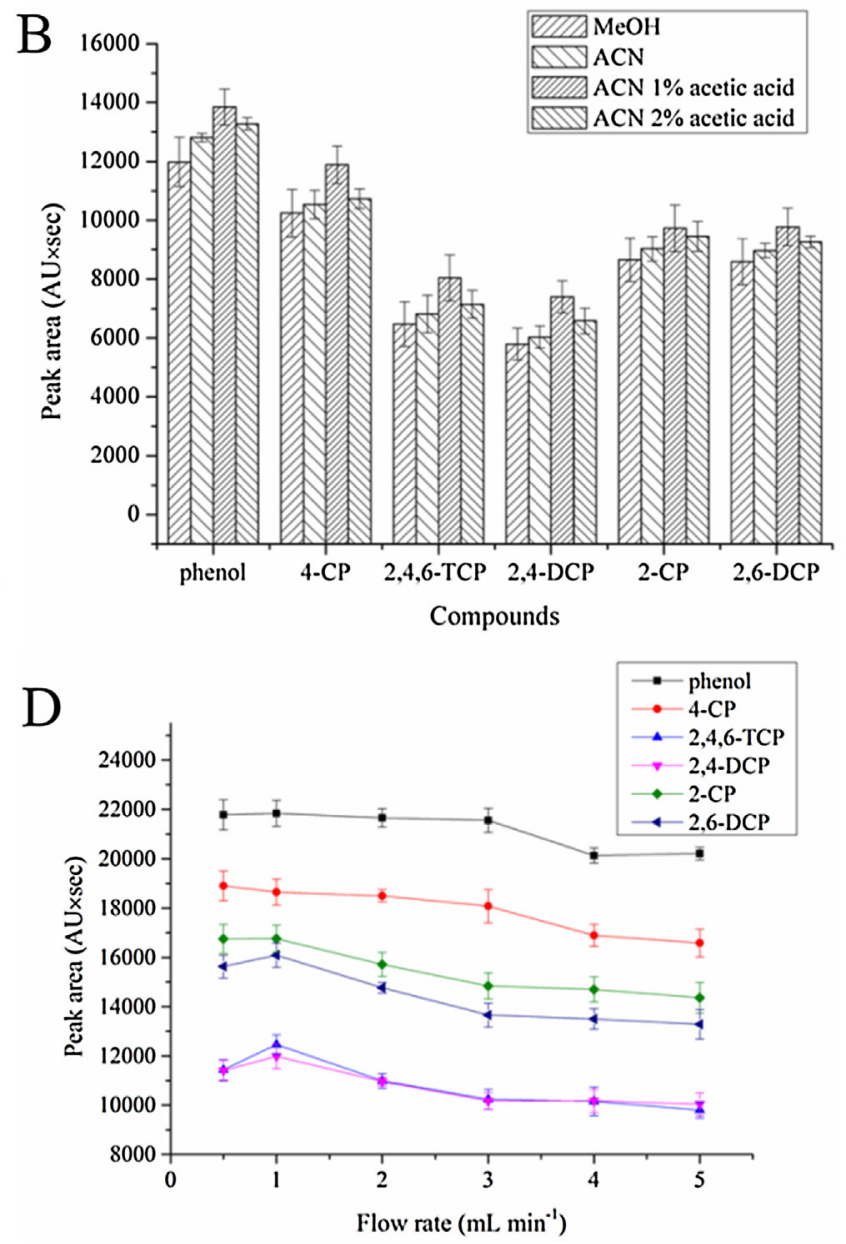

Fig. 4. Optimization of MISPE procedure. Effect of sample pH (A), type of elution solvent (B), volume of elution solvent (C) and flow rate (D).

sample and salt effect. The whole optimization experiments were performed in triplicate using ultrapure water spiked with the six phenolic compounds at $50 \mu \mathrm{g} \mathrm{L}^{-1}$.

Sample $\mathrm{pH}$ was an important factor influencing the form of phenolic compounds and their water solubility because most of phenolic compounds are weak acids. A series of sample $\mathrm{pH}$ values ranging from 3 to 11 by adding varied amounts of $1 \mathrm{~mol} \mathrm{~L}^{-1} \mathrm{HCl}$ or $1 \mathrm{~mol} \mathrm{~L}^{-1} \mathrm{NaOH}$ were investigated. As shown in Fig. 4A, the extraction efficiency of six phenolic compounds increased gradually with sample $\mathrm{pH}$ from 3 to 7 , reached an optimum value at neutral $\mathrm{pH}$ and decreased at basic $\mathrm{pH}$ ranging from 8 to 11 . The possible reason is that most of the phenolic compounds existed in molecular forms at neutral $\mathrm{pH}$, since the $\mathrm{pKa}$ values of phenol, 4-CP, 2-CP, 2,4DCP, 2,4,6-TCP and 2,6-DCP are 9.99, 9.18, 8.56, 7.89, 7.42 and 6.79, respectively [32-34], and thereby they could be strongly rebound to the mt-MIPs. Therefore, neutral condition for sample $\mathrm{pH}=7$ was selected.

Elution is the crucial step in MISPE in order to elute all the analytes retained in the adsorbents. Four kinds of elution solvents including methanol, acetonitrile, acetonitrile containing $1 \%$ acetic acid $(\mathrm{v} / \mathrm{v})$ and acetonitrile containing $2 \%$ acetic acid $(\mathrm{v} / \mathrm{v})$ were investigated. As seen from Fig. 4B, acetonitrile exhibited better elution efficiency than methanol and the addition of acetic acid in acetonitrile could increase the extraction efficiency of phenolic compounds. The free hydrogen of acetic acid could compete with the hydroxyl group of phenolic compounds and form stronger bonding with the functional groups in the binding sites of polymers, which could break the hydrogen bonding interaction between the target analytes and MIPs sorbents. But more acetic acid could influence elution and decrease extraction efficiency [13]. As a result, acetonitrile containing $1 \%$ acetic acid $(v / v)$ was chosen as elution solvent. Besides, the volume of elution solvent was also studied in the range of $100-600 \mu \mathrm{L}$, as shown in Fig. 4C. The extraction efficiency increased fast ranging from 100 to $300 \mu \mathrm{L}$ up to maximum at $400 \mu \mathrm{L}$ and decreased with the volume increasing. Using a low volume of elution solvent, the analytes retained in the MIPs sorbents could not be eluted sufficiently. When the volume increased to $400 \mu \mathrm{L}$, almost all of the analytes could be eluted, and moreover, continuously increasing the volume would reduce the pre-concentration factor. To ensure complete elution and high preconcentration, $400 \mu \mathrm{L}$ acetonitrile containing $1 \%$ acetic acid (v/v) was employed as elution solution.

In order to obtain high extraction efficiency within relatively short time, the effect of sample flow rate should be investigated. $15 \mathrm{~mL}$ aqueous solution containing $50 \mu \mathrm{g} \mathrm{L}^{-1}$ six phenolic compounds was loaded into the MISPE cartridge with the flow rate ranging from 0.5 to $5.0 \mathrm{~mL} \mathrm{~min}^{-1}$, as shown in Fig. $4 \mathrm{D}$. The optimized extraction efficiency was obtained at the flow rate of $1.0 \mathrm{~mL} \mathrm{~min}^{-1}$, while higher rates led to a slight decrease as the contact time between the analytes and MIPs sorbent was decreased. Accordingly, the flow rate of $1.0 \mathrm{~mL} \mathrm{~min}^{-1}$ was selected for the further study.

In general, the addition of salt to the sample can enhance the ionic strength which can decrease the solubility of the analytes. To investigate the effect of salt, different amounts of $\mathrm{NaCl}$ (i.e., 0,3 , $5,10,15 \%(\mathrm{w} / \mathrm{v}))$ were added into the sample solution. The results 
A
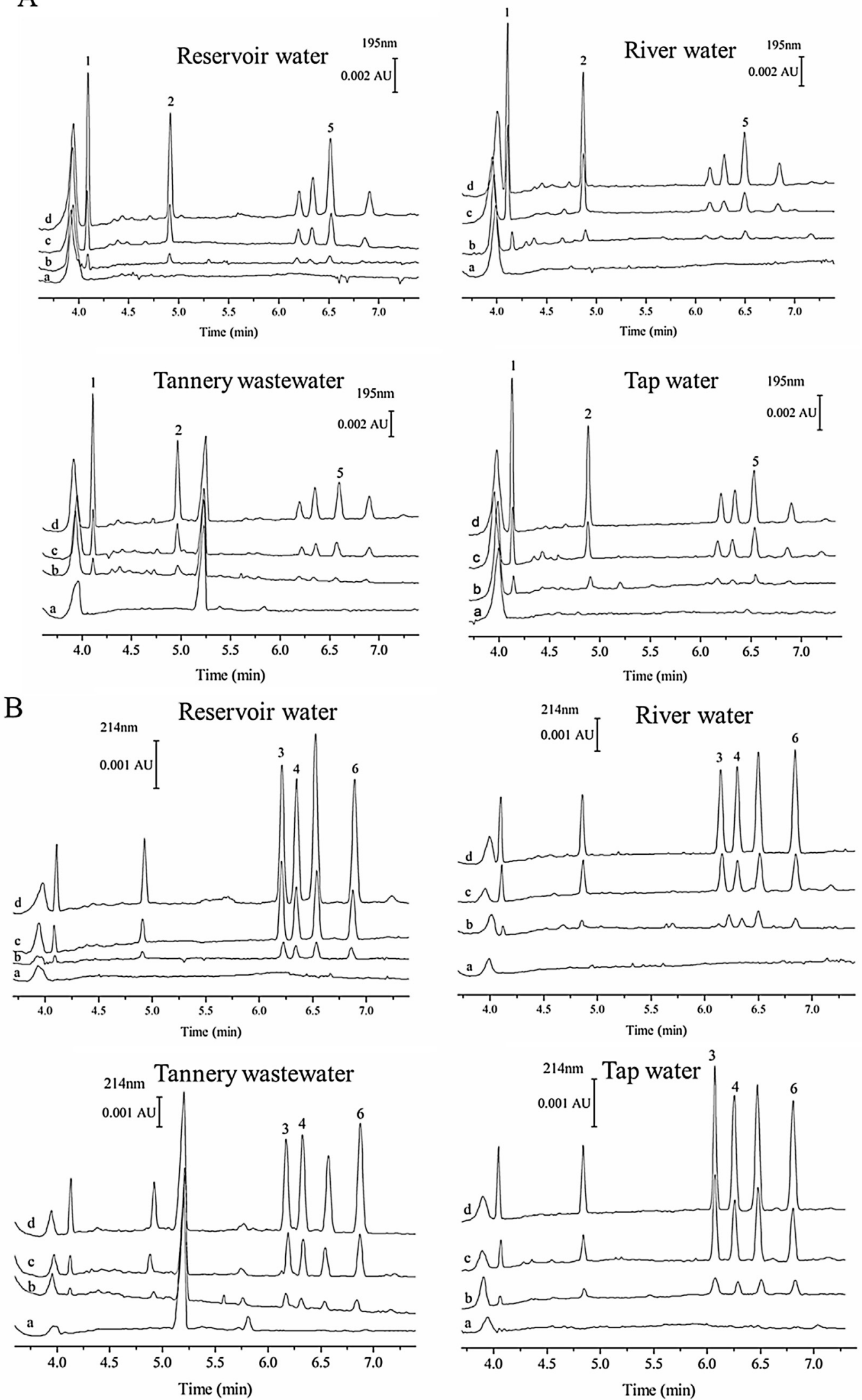

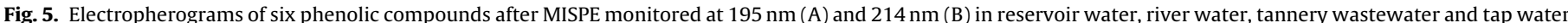

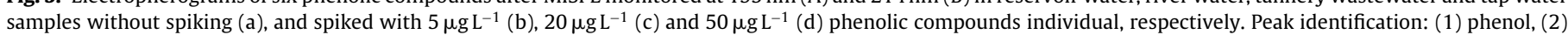

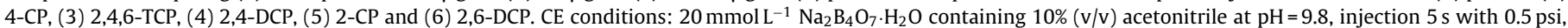
$+20 \mathrm{kV}$ applied voltage. 
Table 1

Analytical performances of the MISPE-CE method for the determination of six phenolic compounds.

\begin{tabular}{|c|c|c|c|c|c|c|c|c|}
\hline Phenolic compounds & $\begin{array}{l}\text { Calibration } \\
\text { equation }{ }^{\mathrm{a}}\end{array}$ & $\begin{array}{l}\text { Correlation } \\
\text { coefficient }(r)\end{array}$ & $\begin{array}{l}\text { Linear range } \\
\left(\mu \mathrm{gL}^{-1}\right)\end{array}$ & $\operatorname{LOD}\left(\mu \mathrm{g} \mathrm{L}^{-1}\right)$ & $\operatorname{LOQ}\left(\mu \mathrm{g} \mathrm{L}^{-1}\right)$ & $\begin{array}{l}\text { Intraday } \\
\text { precision }^{\mathrm{b}}(\mathrm{RSD}, \\
\%)(n=5)\end{array}$ & $\begin{array}{l}\text { Interday } \\
\text { precision }^{\mathrm{b}}(\mathrm{RSD}, \\
\%)(n=5)\end{array}$ & $\mathrm{EF}$ \\
\hline Phenol & $y=306.4 x+243.3$ & 0.9993 & $1-200$ & 0.17 & 0.57 & 4.85 & 5.81 & 55 \\
\hline $4-\mathrm{CP}$ & $y=227.7 x+246.5$ & 0.9960 & $1-200$ & 0.20 & 0.67 & 4.90 & 4.04 & 70 \\
\hline $2,4,6-\mathrm{TCP}$ & $y=151.7 x+599.3$ & 0.9951 & $1-300$ & 0.22 & 0.73 & 2.56 & 3.11 & 118 \\
\hline $2,4-\mathrm{DCP}$ & $y=132.1 x+263.4$ & 0.9986 & $1-300$ & 0.28 & 0.93 & 4.53 & 5.26 & 95 \\
\hline $2-\mathrm{CP}$ & $y=161.0 x+324.3$ & 0.9915 & $1-300$ & 0.25 & 0.83 & 5.51 & 5.05 & 87 \\
\hline 2,6-DCP & $y=167.6 x+45.43$ & 0.9980 & $1-300$ & 0.31 & 1.03 & 2.20 & 3.57 & 85 \\
\hline
\end{tabular}

a $y$ and $x$ stand for the peak area and the concentration $\left(\mu \mathrm{g} \mathrm{L}^{-1}\right)$ of all the analytes, respectively.

b Concentration of $50 \mu \mathrm{g} \mathrm{L}^{-1}$ individual for all the analytes was chosen for the assays of intraday precision and interday precision.

showed that the extraction efficiency decreased with $\mathrm{NaCl}$ increasing, possibly owing to the increase of solution viscosity [35]. Thus, no salt was added for the further experiments.

Consequently, to investige the reusability of the MISPE cartridge, samples $(\mathrm{pH}=7)$ without addition of salt was firstly loaded onto the cartridge at the flow rate of $1.0 \mathrm{~mL} \mathrm{~min}^{-1}$ and eluted with $400 \mu \mathrm{L}$ acetonitrile containing $1 \%$ acetic acid (v/v). Then, the used cartridge was reused for the next MISPE procedure after washing with $3 \mathrm{~mL}$ acetonitrile/acetic acid $=90: 10(\mathrm{v} / \mathrm{v}), 6 \mathrm{~mL}$ acetonitrile and $3 \mathrm{~mL}$ water. The results showed that the MISPE cartridge could be reused at least 20 times without significant template compounds residual, indicating that the prepared PPMIPs had a good stability and reusability.

\subsection{Analytical performances of the MISPE-CE}

Under the optimized MISPE conditions described above, two typical electropherograms of standard solutions of six phenolic compounds at $5 \mathrm{mg} \mathrm{L}^{-1}$ individual without MISPE (curve a) and $50 \mu \mathrm{g} \mathrm{L}^{-1}$ with MISPE (curve b) were attained, as shown in Fig. S3. The analytical performances of our proposed MISPE-CE method were evaluated by the determination of linearity, sensitivity and repeatability, and the excellent results were presented in Table 1. Calibration curves were obtained by plotting the mean peak areas against the concentration of six phenolic compounds in sample solution, and good linearity was attained in the range of $1-200 \mu \mathrm{g} \mathrm{L}^{-1}$ for phenol and 4-CP, $1-300 \mu \mathrm{g} \mathrm{L}^{-1}$ for 2,4,6-TCP, 2,4-DCP, 2-CP and 2,6-DCP, with correlation coefficient $(r)$ higher than 0.9915 . The limits of detection (LODs) were obtained based on the peak height as three times of background noise $(\mathrm{S} / \mathrm{N}=3)$, in the range of $0.17-0.31 \mu \mathrm{g} \mathrm{L}^{-1}$. The limits of quantification (LOQs) calculated based on a signal-to-noise ratio $(\mathrm{S} / \mathrm{N})$ of 10 were in the range of $0.57-1.03 \mu \mathrm{g} \mathrm{L}^{-1}$. The relative standard deviations (RSD) were calculated via five replicate analyses at $50 \mu \mathrm{g} \mathrm{L}^{-1}$ of all the six analytes, and then the intraday and interday precisions were obtained ranging from 2.20 to $5.51 \%$ and 3.11 to $5.81 \%$, respectively. The enrichment factor (EF) was defined as the ratio between the concentrations of analytes before and after MISPE. Then the attained EFs within 55-118 showed relatively high pretreatment efficiency.

\subsection{Applications of the MISPE-CE to real water samples}

In order to evaluate the practical applicability of the validated MISPE-CE method, four real water samples were investigated, including reservoir water, river water, tannery wastewater and tap water samples. Fig. 5 shows the typical electropherograms at the detection wavelength of $195 \mathrm{~nm}$ (A) and $214 \mathrm{~nm}$ (B), respectively. As seen, the endogenous phenolic compounds were not detected in the four water samples (curve a). For the spiked water samples, the six types of phenolic compounds attained complete base-line separation (curves b, c, d). Obviously, the analysis of the phenolic compounds did not suffer from the interference from water matrices, quite probably owing to the selective enrichment ability and good cleanup effect of MISPE. To assess the method performance in complex matrices, the recoveries were investigated by spiking six phenolic compounds at three levels $(5,20$ and $50 \mu \mathrm{g} \mathrm{L}^{-1}$ ) and analyzing five replicates for each concentration. As listed in Table S3, satisfactory recoveries were obtained in the range of $85.57-105.63 \%$ with RSDs of $1.68-6.05 \%$ for reservoir water, $90.91-105.59 \%$ with RSDs of $1.93-6.17 \%$ for river water, 82.13-103.09\% with RSDs of $2.58-6.96 \%$ for tannery wastewater, and $84.84-100.66 \%$ with RSDs of $2.43-6.16 \%$ for tap water. The results indicated that the mt-MIPs based MISPE had a high selectivity to six phenolic compounds and was practically feasible for reliable trace determination in complicated water samples, and consequently phenolic compounds monitoring could be successfully performed.

\section{Conclusions}

In conclusion, the new six-molecule templating mt-MIPs were prepared by a simple precipitation polymerization, and were successfully applied to highly selectively pre-concentrate and extract six kinds of phenolic compounds simultaneously in water samples, followed by CE determination. The present multiple compounds as the template strategy provided novel multi-target adsorption/separation substrates and would bring promising potentials that various concerned species can be imprinted in a single polymer format for high throughput applications. The mt-MIPs based MISPE coupled with CE offered low LODs from 0.17 to $0.31 \mu \mathrm{g} \mathrm{L}^{-1}$, showed satisfactory linearity, precision and accuracy, and was successfully applied to four kinds of real water samples. Consequently, the MISPE-CE method was demonstrated to be a simple, rapid, costeffective and eco-friendly candidate for simultaneous separation and determination of trace phenolic compounds in complicated aqueous matrices. And furthermore, a high throughput analysis MIPs-based platform can be constructed for the efficient monitoring and remediation of water quality, by making more efforts to continuously improve the imprinting performances of mt-MIPs.

\section{Conflict of interest}

The authors declare no competing financial interest.

\section{Acknowledgements}

This work was financially supported by the Strategic Priority Research Program of the Chinese Academy of Sciences (XDA11020702), the National Defense Science and Technology Innovation Project of Chinese Academy of Sciences (CXJJ-16M254), the Ministry of Science and Technology Project Foundation (2014FY210600) and the National Natural Science Foundation of China (21477160, 21275158). 


\section{Appendix A. Supplementary data}

Supplementary data associated with this article can be found, in the online version, at http://dx.doi.org/10.1016/j.chroma.2016.12. 069.

\section{References}

[1] R. Alizadeh, P.K. Kashkoei, M. Kazemipour, Zinc oxide-copper oxide nanoplates composite as coating for solid phase microextraction combined with high performance liquid chromatography-UV detection for trace analysis of chlorophenols in water and tomato juice samples, Anal. Bioanal. Chem. 408 (2016) 3727-3736

[2] M.S. Noorashikin, A.B. Nur Nadiah, I. Nurain, A.A. Siti Aisyah, M.R. Siti Zulaika, Determination of phenol in water samples using cloud point extraction and UV spectrophotometry, Desalin. Water Treat. 57 (2016) 15486-15494.

[3] R.G. Dolatto, I. Messerschmidt, B. Fraga Pereira, R. Martinazzo, G. Abate, Preconcentration of polar phenolic compounds from water samples and soil extract by liquid-phase microextraction and determination via liquid chromatography with ultraviolet detection, Talanta 148 (2016) 292-300.

[4] A. González, J. Avivar, V. Cerdà, Determination of priority phenolic pollutants exploiting an in-syringe dispersive liquid-liquid microextraction-multisyringe chromatography system, Anal. Bioanal. Chem. 407 (2015) 2013-2022.

[5] J.A. Padilla-Sánchez, P. Plaza-Bolaños, R. Romero-González, N. Barco-Bonilla, J.L. Martínez-Vidal, A. Garrido-Frenich, Simultaneous analysis of chlorophenols, alkylphenols, nitrophenols and cresols in wastewater effluents, using solid phase extraction and further determination by gas chromatography-tandem mass spectrometry, Talanta 85 (2011) 2397-2404

[6] Z. Lin, Z. Zhang, Y. Li, Y. Deng, Recyclable magnetic-pickering emulsion liquid membrane for extracting phenol compounds from wastewater, J. Mater. Sci. 51 (2016) 6370-6378.

[7] M.M. Abolghasemi, S. Parastari, V. Yousefi, A nanoporous anodized alumina wire with a nanosized hydroxyapatite coating for headspace solid-phase microextraction of phenol and chlorophenols, Microchim. Acta 183 (2016) 241-247.

[8] C. Zhong, M. He, H. Liao, B. Chen, C. Wang, B. Hu, Polydimethylsiloxane/covalent triazine frameworks coated stir bar sorptive extraction coupled with high performance liquid chromatography-ultraviolet detection for the determination of phenols in environmental water samples, J. Chromatogr. A 1441 (2016) 8-15.

[9] Y. Wen, L. Chen, J. Li, D. Liu, L. Chen, Recent advances in solid-phase sorbents for sample preparation prior to chromatographic analysis, TrAC-Trends Anal. Chem. 59 (2014) 26-41.

[10] L. Chen, S. Xu, J. Li, Recent advances in molecular imprinting technology: current status, challenges and highlighted applications, Chem. Soc. Rev. 40 (2011) 2922-2942.

[11] L. Chen, X. Wang, W. Lu, X. Wu, J. Li, Molecular imprinting: perspectives and applications, Chem. Soc. Rev. 45 (2016) 2137-2211.

[12] A.H. El-Sheikh, R.W. Al-Quse, M.I. El-Barghouthi, F.S. Al-Masri, Derivatization of 2-chlorophenol with 4-amino-anti-pyrine: a novel method for improving the selectivity of molecularly imprinted solid phase extraction of 2-chlorophenol from water, Talanta 83 (2010) 667-673.

[13] H. Surikumaran, S. Mohamad, N. Muhamad Sarih, R. Muggundha Raoov, $\beta$-Cyclodextrin based molecular imprinted solid phase extraction for class selective extraction of priority phenols in water samples, Sep. Sci. Technol. 50 (2015) 2342-2351.

[14] Q. Feng, L. Zhao, J.M. Lin, Molecularly imprinted polymer as micro-solid phase extraction combined with high performance liquid chromatography to determine phenolic compounds in environmental water samples, Anal. Chim. Acta 650 (2009) 70-76.

[15] A.C. Meng, J. LeJeune, D.A. Spivak, Multi-analyte imprinting capability of OMNiMIPs versus traditional molecularly imprinted polymers, J. Mo Recognit. 22 (2009) 121-128.

[16] X.L. Song, J.H. Li, S.F. Xu, R.J. Ying, J.P. Ma, C.Y. Liao, D.Y. Liu, J.B. Yu, L.X. Chen, Determination of 16 polycyclic aromatic hydrocarbons in seawater using molecularly imprinted solid-phase extraction coupled with gas chromatography-mass spectrometry, Talanta 99 (2012) 75-82.
[17] M. Lombardo-Agui, A.M. Garcia-Campana, L. Gamiz-Gracia, C.C. Blanco, Laser induced fluorescence coupled to capillary electrophoresis for the determination of fluoroquinolones in foods of animal origin using molecularly imprinted polymers, J. Chromatogr. A 1217 (2010) 2237-2242.

[18] S.R. Mei, D. Wu, M. Jiang, B. Lu, J.M. Lim, Y.K. Zhou, Y.I. Lee, Determination of trace bisphenol A in complex samples using selective molecularly imprinted solid-phase extraction coupled with capillary electrophoresis, Microchem. J. 98 (2011) 150-155.

[19] H.W. Wang, Y.Q. Liu, S.L. Wei, S. Yao, J.L. Zhang, H.C. Huang, Selective extraction and determination of fluoroquinolones in bovine milk samples with montmorillonite magnetic molecularly imprinted polymers and capillary electrophoresis, Anal. Bioanal. Chem. 408 (2016) 589-598.

[20] J. Pan, X. Zou, X. Wang, W. Guan, Y. Yan, J. Han, Selective recognition of 2,4-dichlorophenol from aqueous solution by uniformly sized molecularly imprinted microspheres with $\beta$-cyclodextrin/attapulgite composites as support, Chem. Eng. J. 162 (2010) 910-918.

[21] J. Wang, P.A. Cormack, D.C. Sherrington, E. Khoshdel, Monodisperse, molecularly imprinted polymer microspheres prepared by precipitation polymerization for affinity separation applications, Angew. Chem. Int. Ed. 42 (2003) 5336-5338

[22] H. Surikumaran, S. Mohamad, N.M. Sarih, Synthesis and evaluation of methacrylic acid functionalized beta-cyclodextrin based molecular imprinted polymer for 2,4-dichlorophenol in water samples, Desalin. Water Treat. 57 (2016) 254-267

[23] M. Yang, A. Han, J. Duan, Z. Li, Y. Lai, J. Zhan, Magnetic nanoparticles and quantum dots co-loaded imprinted matrix for pentachlorophenol, J. Hazard. Mater. 237-238 (2012) 63-70.

[24] J. Yang, J. Li, J. Qiao, S. Cui, H. Lian, H. Chen, Magnetic solid phase extraction of brominated flame retardants and pentachlorophenol from environmental waters with carbon doped $\mathrm{Fe}_{3} \mathrm{O}_{4}$ nanoparticles, Appl. Surf. Sci. 321 (2014) $126-135$.

[25] K. Yoshimatsu, K. Reimhult, A. Krozer, K. Mosbach, K. Sode, L. Ye, Uniform molecularly imprinted microspheres and nanoparticles prepared by precipitation polymerization: the control of particle size suitable for different analytical applications, Anal. Chim. Acta 584 (2007) 112-121.

[26] X. Wei, Z. Zhou, T. Hao, H. Li, Y. Yan, Molecularly imprinted polymer nanospheres based on Mn-doped ZnS QDs via precipitation polymerization for room-temperature phosphorescence probing of 2,6-dichlorophenol, RSC Adv. 5 (2015) 19799-19806.

[27] Z. Zhang, S. Xu, J. Li, H. Xiong, H. Peng, L. Chen, Selective solid-phase extraction of Sudan I in chilli sauce by single-hole hollow molecularly imprinted polymers, J. Agric. Food Chem. 60 (2012) 180-187.

[28] Z. Zhang, L. Chen, F. Yang, J. Li, Uniform core-shell molecularly imprinted polymers: a correlation study between shell thickness and binding capacity, RSC Adv. 4 (2014) 31507-31514.

[29] W. Guan, C. Han, X. Wang X. Zou, J. Pan, P. Huo, C. Li, Molecularly imprinted polymer surfaces as solid-phase extraction sorbents for the extraction of 2-nitrophenol and isomers from environmental water, J. Sep. Sci. 35 (2012) 490-497.

[30] F. An, B. Gao, X. Feng, Adsorption and recognizing ability of molecular imprinted polymer MIP-PEI/SiO 2 towards phenol, J. Hazard. Mater. 157 (2008) 286-292.

[31] J. Dong, H. Fan, D. Sui, L. Li, T. Sun, Sampling 4-chlorophenol in water by DGT technique with molecularly imprinted polymer as binding agent and nylon membrane as diffusive layer, Anal. Chim. Acta 822 (2014) 69-77.

[32] C. Baggiani, L. Anfossi, C. Giovannoli, C. Tozzi, Multivariate analysis of the selectivity for a pentachlorophenol-imprinted polymer, J. Chromatogr. B 804 (2004) 31-41.

[33] I. Rodríguez, M.P. Llompart, R. Cela, Solid-phase extraction of phenols, J Chromatogr. A 885 (2000) 291-304

[34] Q. Wang, H. Qiu, J. Li, X. Liu, S. Jiang, On-line coupling of ionic liquid-based single-drop microextraction with capillary electrophoresis for sensitive detection of phenols, J. Chromatogr. A 1217 (2010) 5434-5439.

[35] X. Liu, X. Zhou, C. Wang, Q. Wu, Z. Wang, Magnetic three-dimensional graphene solid-phase extraction of chlorophenols from honey samples, Food Addit. Contam. A 32 (2015) 40-47. 